\title{
A retrospective study on prophylactic regional lymphadenectomy versus nodal observation only in the management of dogs with stage I, completely resected, low-grade cutaneous mast cell tumors
}

Silvia Sabattini ${ }^{1}$, Matti Kiupel ${ }^{2}$, Riccardo Finotello ${ }^{3}$, Damiano Stefanello ${ }^{4}$, Eugenio Faroni ${ }^{1}$, Walter Bertazzolo ${ }^{5}$, Ugo Bonfanti ${ }^{5}$, Antonella Rigillo', Sara Del Magno ${ }^{1}$, Armando Foglia', Luca Aresu', Matteo Gambini ${ }^{4}$, Mario Caniatti ${ }^{4}$ and Laura Marconato ${ }^{{ }^{*}}$

\begin{abstract}
Background: While lymphadenectomy of metastatic lymph nodes (LNs) has been associated with improved outcome, the clinical utility of prophylactic lymphadenectomy in dogs with stage I cutaneous mast cell tumors (CMCTs) remains a controversial topic. To assess the therapeutic role of lymphadenectomy of uninvolved regional LNs, the long-term outcome of CMCT-bearing dogs with cytologically negative and surgically unresected regional LNs (observation only, OO) was compared with that of dogs with surgically resected and histologically negative regional LNs (prophylactic regional lymphadenectomy, PRL).

Results: A retrospective analysis of 64 dogs with a low-grade, completely resected stage I CMCT was performed: 35 (54.7\%) dogs were subjected to $\mathrm{OO}$ and 29 (45.3\%) underwent PRL. Dogs were monitored for a median of 813 and 763 days in the $\mathrm{OO}$ group and PRL group, respectively. The number of dogs undergoing MCT progression was significantly higher in the $\mathrm{OO}$ group $(P=0.028)$ and curve comparison revealed a tendency to a better time to progression in the PRL group $(P=0.058)$. No significant difference in survival time $(P=0.294)$ was observed between dogs in the $\mathrm{OO}$ and PRL groups.

Conclusions: Our results showed that lack of immediate lymphadenectomy was associated with a higher risk for tumor progression. This preliminary judgement, reinforced by the findings that lymphadenectomy was well tolerated in all cases, and that histopathology provides the definitive assessment of the nodal pathological status, may suggest that prophylactic lymphadenectomy is indicated in the management of stage I MCTs. Larger prospective studies are warranted for generating clinical evidence of this latter hypothesis.
\end{abstract}

Keywords: Canine, Lymphadenectomy, Mast cell tumor, Observation, Stage I

*Correspondence: laura.marconato@unibo.it

1 Department of Veterinary Medical Sciences, University of Bologna,

Ozzano dell'Emilia, Bologna, Italy

Full list of author information is available at the end of the article

\section{Background}

In canine cutaneous mast cell tumors (cMCTs), lymphatic drainage from the primary tumor has long been recognized as the most common initial route of 
metastatic spread, with the first site of metastasis identified as the draining nodal basin [1-3].

Prophylactic lymphadenectomy refers to the complete dissection of lymph nodes in human and veterinary patients with no evidence of nodal involvement [4-6]. While the therapeutic effect of regional lymphadenectomy has been documented in dogs with stage II cMCTs, the role of prophylactic regional lymphadenectomy in animals with stage I disease remains a controversial topic [7]. More often, dogs present with a clinically non aggressive cMCT and a cytologically negative lymph node (LN). It is difficult to advise owners about the need to surgically remove the $\mathrm{LN}$ alongside the primary tumor, as the amount and quality of information currently available does not offer a definitive answer to the question of the prognostic effect of prophylactic regional lymphadenectomy in early stage cMCTs.

Both an elective lymphadenectomy and a watchfulwaiting policy have their proponents. The suspected high incidence rate of undetected or late LN metastasis in cMCTs is the main argument in favor of prophylactic lymphadenectomy, which is based on the rationale that further metastatic spread could be prevented at the level of the regional LN by eliminating the potential first neoplastic reservoir [8].

Conversely, the main arguments against prophylactic lymphadenectomy include the morbidities from the procedure including risk of lymphedema, increased length of surgery and complications from wound healing with unclear benefit, and the interference with the protective immune response to metastatic disease by removal of unaffected regional LNs $[9,10]$. Such evidence derives from human medicine only, where patients undergo massive nodal dissection and immunity studies have been performed.

Additionally, the recent introduction of sentinel LN mapping in the diagnostic work-up of cMCTs has introduced the question regarding the clinical usefulness of prophylactic regional lymphadenectomy. According to three recent studies, the sentinel LN was different to the regional LN in $25-60 \%$ of dogs with cMCT $[3,11,12]$.

Another problem of utmost importance concerning prophylactic lymphadenectomy indication is related to the diagnostic methods to classify a dog as node-positive or negative. The clinical examination, upon which the WHO classification is based, is far from being accurate, as palpation as well as imaging studies are unreliable predictors of nodal metastasis by themselves $[13,14]$.

It has been recently shown that non-palpable or normal-sized LNs may harbor metastatic disease and approximately $50 \%$ of those nodes either had early metastatic (HN2 according to the Weishaar classification) or overtly metastatic (HN3) disease, whereas the other half of dogs had nodes with no evidence of metastatic disease (HNO) or minimal suspicion of metastasis (HN1) $[8,15]$.

Due to the above, cytologic evaluation of the regional LN is always advised for the assessment of metastatic involvement [1]. Fine-needle aspiration (FNA) of the regional $\mathrm{LN}$ has been established as a cost-effective diagnostic tool to screen dogs for metastatic disease [16, 17].

Until 2009, the reporting and interpretation of LN cytology had caused considerable confusion in comparing results from different settings [18]. The introduction of the Krick criteria provided the opportunity to establish standard terminology and reporting guidelines for different diagnostic categories [16]. Based on cytology, five categories associated with escalating risk of malignancy have been proposed: "normal LNs", "hyperplastic LNs", "possible", "probable" and "certain" metastasis based on the number of mast cells and the number and size of mast cell aggregates [16]. However, not unexpectedly, cytologic diagnosis of nodal metastasis may yield falsepositive or false-negative results, leading to $>25 \%$ of discrepant cases when cytology and histology are compared $[17,19]$.

Another classification has been proposed by Weishaar et al. to standardize the histological assessment of metastatic involvement in dogs with cMCTs [15]. This system was found to correlate with clinical outcome in the original study [15]. However, labeling of the categories HN1 and HN2 is misleading, as they indicate stages of disease progression rather than degrees of a suspected diagnosis. Furthermore, the system is not based on a standardized trimming approach of examined nodes that may result in similar high false-positive and false-negative results as the cytologic system $[15,20,21]$. Regardless, the question that arises is whether LNs with no to rare (0-3), scattered, individualized mast cells in sinuses and/or parenchyma (HNO) or greater than three individualized mast cells in sinuses and/or parenchyma in a minimum of four highpower fields (HN1) represent no metastatic disease or whether dogs with such nodes will go on to develop macroscopic disease, stressing a different biology at play [22]. If $\mathrm{HNO} / \mathrm{HN} 1 \mathrm{LNs}$ are essentially clinically and prognostically insignificant, lymphadenectomy could be targeted to only those LNs harboring metastatic disease (HN2/ HN3). This would, however, require accurate determination of nodal metastasis without lymphadenectomy.

Thus, to investigate whether removal of potential clinically occult metastatic disease is associated with improved outcome, we first carried out an agreement study aimed at assessing the concordance between Krick's cytological classification and Weishaar's histological classification in diagnosing a LN as non-metastatic. Then, we retrospectively compared dogs with stage I, completely resected, low-grade cMCTs undergoing 
prophylactic regional lymphadenectomy (PRL group) with those where the regional LN was only monitored over time (observation only group, OO group).

We hypothesized that PRL provides a clinical benefit and is well tolerated.

Informed consent was obtained from animal owners for using data for the research purpose. Since this was a retrospective study, no approval from the Ethical Committee was required.

\section{Results}

\section{Agreement study}

Eighty-two cMCT-bearing dogs with cytologically negative regional LN undergoing subsequent lymphadenectomy and histological examination were reviewed: 48 (58.5\%) LN aspirates were interpreted as normal and $34(41.5 \%)$ as reactive according to Krick's cytological evaluation. On the original histopathology reports, 48 (58.5\%) LNs were interpreted as non-metastatic (HNO), $30(36.6 \%)$ as pre-metastatic (HN1) and $4(4.9 \%)$ as early metastatic (HN2). The negative predictive value of cytology in the identification of cMCT nodal metastases was $95.1 \%$. This was considered sufficient to confirm the reliability of cytology in the identification of dogs without LN metastasis and to perform the subsequent clinical study.

\section{Clinical study}

\section{Patients and tumor characteristics}

Overall, 64 dogs were included in the analysis. The most represented breeds were Labrador retriever $(n=17$, $26.5 \%)$, Boxer $(n=10,15.6 \%)$ and American Staffordshire terrier $(n=4,11.4 \%)$. Of the remaining dogs, 10 were mixed-breed dogs, and 23 were breeds that were represented once or twice.

Median age was 7 years (range, 1-13) and median weight was $30.7 \mathrm{~kg}$ (range, 5-55). There were 36 females (29 spayed) and 28 males (15 neutered).

Tumors were located on limbs $(n=27 ; 42.2 \%)$, head and neck $(n=16 ; 25 \%)$, trunk $(n=13 ; 20.3 \%)$, inguinal region $(n=5 ; 7.8 \%)$ and mammary region $(n=3 ; 4.7 \%)$. Median maximum tumor diameter was $1.3 \mathrm{~cm}$ (range, 0.3-5.4); 60 (93.7\%) cMCTs were not ulcerated, while 4 (6.3\%) were.

All dogs were asymptomatic at presentation.

Based on the Patnaik grading system, there were 8 (14.3\%) grade 1 cMCTs, and 56 (85.7\%) grade 2 cMCTs. All were Kiupel low-grade.

Twenty-nine (45.3\%) dogs had undergone PRL, whereas 35 (54.7\%) had been subjected to OO.

In the PRL group, the following LNs were removed: inguinal $(n=10 ; 34.5 \%)$, superficial cervical $(n=7$; $24.1 \%)$, submandibular $(n=6 ; 20.7 \%)$, popliteal $(n=5$; $17.2 \%)$ and axillary $(\mathrm{n}=1,3.4 \%)$. Among them, 19
(65.5\%) LNs were interpreted as non-metastatic (HNO) and $10(34.5 \%)$ as pre-metastatic (HN1).

In the OO group, the following LNs were sampled: superficial cervical $(n=13 ; 37.1 \%)$, inguinal $(n=9$; $25.7 \%)$, popliteal $(n=5,14.3 \%)$, axillary $(n=4,11.4 \%)$, and submandibular $(\mathrm{n}=4,11.4 \%)$. Among them, 27 (77.1\%) LN aspirates were interpreted as normal and 8 (22.9\%) as reactive.

The only difference among groups regarding demographic features and possible prognostic variables was a tendency towards a proportion of breeds predisposed to low-grade cMCTs in the OO group (Table 1).

\section{Treatment and outcome}

In the OO group, the median follow-up time was 813 days (range, 290-2900). Overall, 6 dogs (17.1\%) experienced cMCT progression after a median of 822 days (range, 560-1380): 4 (11.4\%) experienced local relapse, $2(5.7 \%)$ experienced nodal metastases in the LNs that had been previously aspirated, and 1 (2.9\%) developed visceral metastasis. Median TTP was not reached. Twelve (34.3\%) dogs developed new cMCTs after a median of 734 days (range, 197-1409) at the following locations: trunk $(n=6)$, limbs $(n=5)$, head and neck $(n=1)$.

At the end of the study, 25 (71.4\%) dogs were alive, 7 (20\%) had died because of cMCT-unrelated causes $(n=3$ cardiac failure, $n=1$ adrenal carcinoma, $n=1$ acute lymphoblastic leukemia; $\mathrm{n}=1$ splenic hemangiosarcoma; $\mathrm{n}=1$ mesothelioma), and $3(8.6 \%)$ had died because of cMCT-related causes after 1215, 1300 and 1471 days. Median ST was not reached.

In the PRL group, surgical complications related to lymphadenectomy did not occur, and no longer hospitalization was required compared with dogs undergoing surgical resection of the primary tumor only. The median follow-up time was 763 days (range, 181-2039). None experienced cMCT progression. Three dogs (10.3\%) developed de novo cMCTs at the trunk after 321, 417 and 1092 days.

At the end of the follow-up period, 28 (96.6\%) dogs were alive, and 1 (3.4\%) had died because of tumorunrelated causes after 835 days (metastatic anal sac carcinoma).

The number of dogs undergoing cMCT progression was significantly higher in the OO group $(P=0.028)$, although no significant difference was observed in TTP $(P=0.058$; Fig. 1 ; Table 1). Similarly, the number of dogs developing new cMCTs was significantly higher in the OO group $(P=0.037$; Table 1$)$.

No significant difference in ST $(P=0.294)$ was observed between dogs in the $\mathrm{OO}$ and PRL groups (Fig. 2). 
Table 1 Demographic information, distribution of variables potentially associated with prognosis and follow-up information of 64 dogs with stage I low grade mast cell tumor treated by surgical excision and prophylactic regional lymphadenectomy or nodal observation only. Differences in data distribution were assessed with Chi-square test/Fisher's exact test (categorical variables) or MannWhitney $U$ test (quantitative variables)

\begin{tabular}{|c|c|c|c|}
\hline Variable & Observation only $(n=35)$ & $\begin{array}{l}\text { Prophylactic regional } \\
\text { lymphadenectomy } \\
(n=29)\end{array}$ & $P$ \\
\hline Purebred & & & 0.164 \\
\hline Yes & 32 & 22 & \\
\hline No & 3 & 7 & \\
\hline Breed & & & 0.078 \\
\hline $\begin{array}{l}\text { Boxer, French bulldog, Weimaraner, Pug, American } \\
\text { Staffordishire terrier }\end{array}$ & 13 & 5 & \\
\hline Other & 22 & 24 & \\
\hline Age (years) & & & 0.224 \\
\hline Median (range) & $6.0(2.0-11.0)$ & $7.0(1.0-13.0)$ & \\
\hline Weight (kg) & & & 0.237 \\
\hline Median (range) & $33.0(8.4-50.4)$ & $27.4(5.0-55.0)$ & \\
\hline Sex & & & 0.454 \\
\hline Male & 17 & 11 & \\
\hline Female & 18 & 18 & \\
\hline Neutering status & & & 0.175 \\
\hline Yes & 27 & 17 & \\
\hline No & 8 & 12 & \\
\hline Anatomic location & & & 0.137 \\
\hline Head and neck & 8 & 8 & \\
\hline Trunk and limbs & 25 & 15 & \\
\hline Inguinal/perineal/mammary/digital & 2 & 6 & \\
\hline Tumor diameter (cm) & & & 0.567 \\
\hline Median (range) & $1.4(0.5-5.4)$ & $1.3(0.3-9.0)$ & \\
\hline Ulceration & & & $>0.999$ \\
\hline Yes & 2 & 2 & \\
\hline No & 33 & 27 & \\
\hline $\begin{array}{l}\text { Follow-up time (days) } \\
\text { Median (range) }\end{array}$ & $813(290-2900)$ & 763 (181-2039) & 0.267 \\
\hline Disease progression & & & $0.028^{*}$ \\
\hline Yes & 6 & 0 & \\
\hline No & 29 & 29 & \\
\hline Development of new MCT & & & $0.037^{*}$ \\
\hline Yes & 12 & 3 & \\
\hline No & 23 & 26 & \\
\hline MCT related death & & & 0.245 \\
\hline Yes & 3 & 0 & \\
\hline No & 32 & 29 & \\
\hline
\end{tabular}

On Cox proportional hazards regression analysis, no factor was significantly associated with an increased risk of cMCT progression or cMCT-related death (Table 2).

cMCT progression and cMCT-related death were not affected by Krick cytological LN score (normal or reactive) in the OO group $(P=0.46$ and $P=0.838$, respectively), or by Weishaar histological LN score (HN0 or
HN1) in the PRL group (analysis not performed due to absence of events).

\section{Discussion}

Over the past decade, new treatments for canine cMCTs have been developed [23]. Nevertheless, it is the authors' opinion that the significant uncertainty in staging 


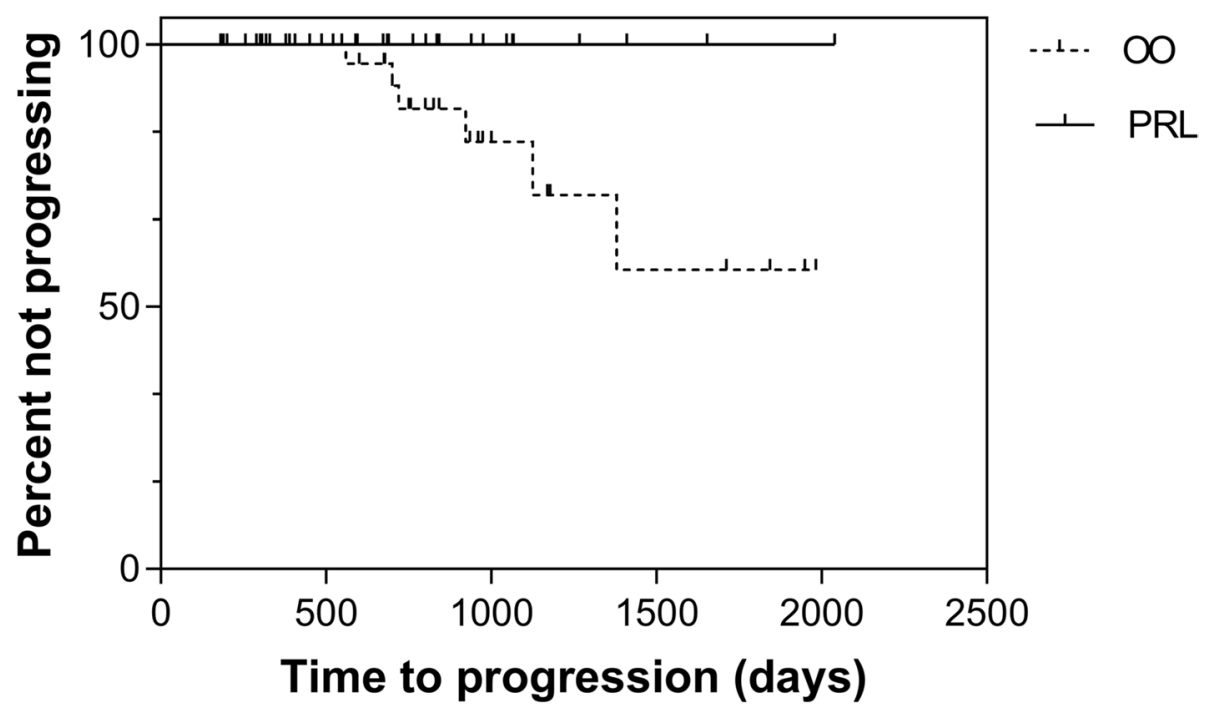

No. at risk

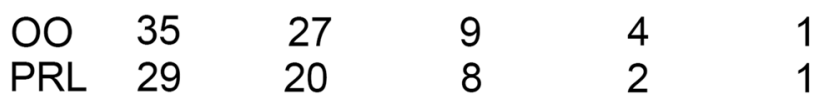

Fig. 1 Time to progression for 64 dogs with surgically-removed stage I low grade mast cell tumor undergoing prophylactic regional lymphadenectomy (PRL, solid line) or regional lymph node observation only (OO, dashed line). Difference not statistically significant $(P=0.058)$

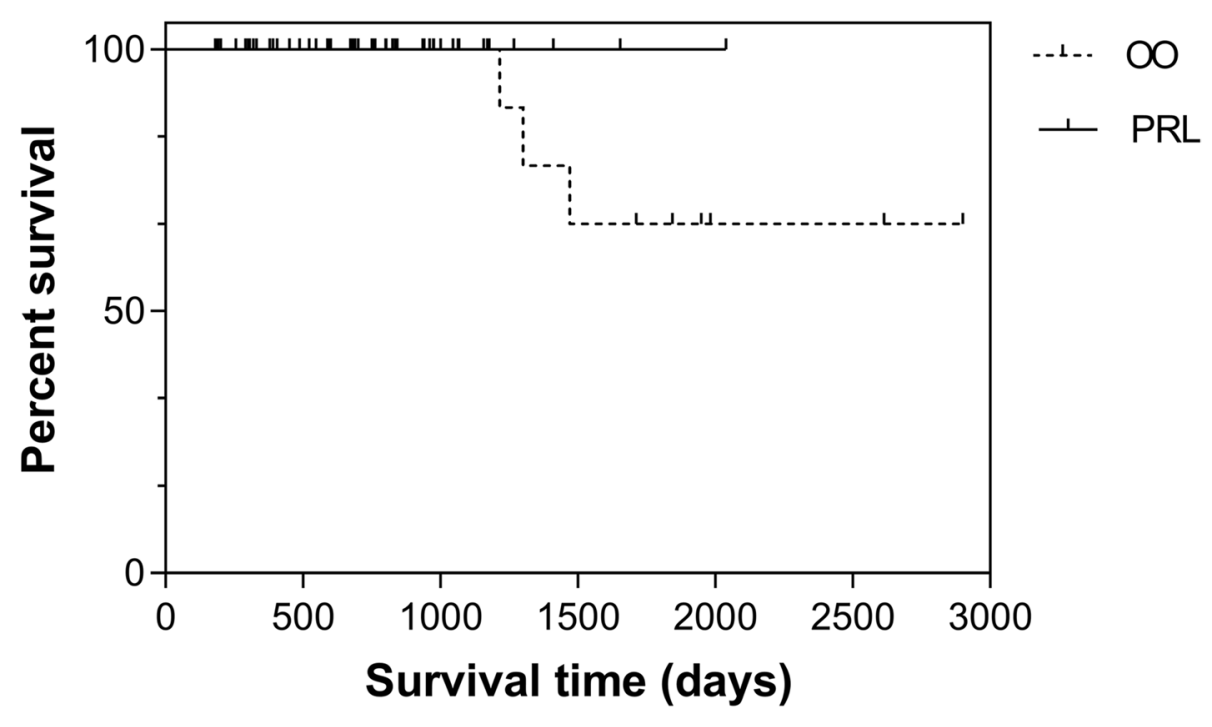

No. at risk

$\begin{array}{llccccc}\mathrm{OO} & 35 & 27 & 14 & 6 & 2 & 2 \\ \mathrm{PRL} & 29 & 20 & 8 & 2 & 1 & 0\end{array}$

Fig. 2 Survival time for 64 dogs with surgically-removed stage I low grade mast cell tumor undergoing prophylactic regional lymphadenectomy (PRL, solid line) or regional lymph node observation only (OO, dashed line). Difference not statistically significant $(P=0.294)$ 
Table 2 Univariable Cox regression analysis of variables potentially associated with increased risk of tumor progression and tumorrelated death in 64 dogs with low-grade, completely resected stage I CMCT

\begin{tabular}{|c|c|c|c|c|}
\hline \multirow[t]{2}{*}{ Variable } & \multicolumn{2}{|l|}{ Tumor progression } & \multicolumn{2}{|l|}{ Tumor-related death } \\
\hline & $\mathrm{HR}(95 \% \mathrm{Cl})$ & $P$ & $\mathrm{HR}(95 \% \mathrm{Cl})$ & $P$ \\
\hline Breed predisposed to biologically aggressive $\mathrm{CMCTs}$ & $0.92(0.16-5.4)$ & 0.925 & $0.39(0.03-4.39)$ & 0.443 \\
\hline Age $>7$ years & $4.02(0.75-21.44)$ & 0.104 & $6.3(0.57-69.79)$ & 0.133 \\
\hline Weight $>30.7 \mathrm{~kg}$ & $1.38(0.25-7.71)$ & 0.714 & $0.99(0.87-1.12)$ & 0.832 \\
\hline Male sex & $0.66(0.12-3.62)$ & 0.634 & $0.7(0.06-7.81)$ & 0.770 \\
\hline Neutering status & $0.42(0.08-2.07)$ & 0.285 & $0.18(0.02-2.01)$ & 0.163 \\
\hline Biologically aggressive anatomic location & $1.51(0.18-13.01)$ & 0.705 & NA & \\
\hline Tumor diameter $>1.3 \mathrm{~cm}$ & $0.56(0.10-3.09)$ & 0.510 & $2.41(0.22-26.59)$ & 0.473 \\
\hline Ulceration & $2.71(0.29-25.30)$ & 0.383 & NA & \\
\hline Patnaik grade 2 & $0.41(0.05-3.59)$ & 0.421 & $0.35(0.03-3.88)$ & 0.39 \\
\hline Lack of prophylactic regional lymphadenectomy & $43.02(0.04-41,697.1)$ & 0.284 & $33.17(0-1,727,740.46)$ & 0.527 \\
\hline
\end{tabular}

work-up and the considerable variability in current practice, mainly due to the lack of prospective evidence, have led to the unstandardized management of locoregional disease. While lymphadenectomy is the current standard approach for clinically suspected or positive LNs, regardless of histological grade of the primary tumor [7, 24], whether clinically unaffected LNs should undergo prophylactic regional lymphadenectomy when the primary cMCT is resected or whether only the primary cMCT should be resected remains a dilemma. Our results overall showed no significant differences in ST between operated dogs and those undergoing OO. However, a significantly higher proportion of dogs developing tumor progression and new cMCTs was observed in the group of dogs not receiving an elective regional LN dissection as part of their primary therapy.

As a general rule, an accurate preoperative diagnosis and strict follow-up are required to provide an adequate surgical dose while ensuring the therapeutic effect by narrowing down the target based on the risk-benefit balance. In other words, when it comes to surgical management, based on the current evidence, the extent of LN dissection should be adapted to clinical stage, as this corresponds to metastatic spread. To do so, several critical aspects need to be taken into consideration.

First, the identification of pathologically negative LNs contributes to the problem. Peripheral LNs are initially evaluated by means of physical examination and cytology, and a high degree of inaccuracy for these methods has been documented in the literature $[8,13,19]$. While the ultimate goal of FNA is to obtain cytologic material sufficiently to render a diagnosis of metastatic or non-metastatic LNs confidently, based on the current literature, the proportion of cytologically negative, histologically positive cases ranges from 10 to $50 \%[8,17,19]$.
In the current study, 82 cytologically negative nodes from the same institutions with the available corresponding histological reports on the surgical sample were retrospectively reviewed, yielding a false negative rate of approximately $5 \%$. Also, $37 \%$ of the LNs were histologically pre-metastatic and not identified by cytology, underscoring the limits of this analysis.

Second, the indications for PRL remain subjects of much debate, since there are widely divergent views concerning the efficacy of routine lymphadenectomy and no evidence-based guidelines.

The argument in favor of PRL is based on the possibility that clinically or even histologically normal nodes may contain isolated malignant cells which, if not removed, may worsen outcome [7]. It is hypothesized that neoplastic cells may lie dormant in the regional LN for a considerable amount of time, only to recur or spread at a later point. This phenomenon has been well documented in human patients with melanoma, and PRL of the sentinel $\mathrm{LN}$ is recommended for selected patients $[25,26]$.

Conversely, removing LNs that appear unaffected may be unnecessary and potentially harmful, considering the higher morbidity associated with the procedure, increased duration of surgery and costs. Also, considering the host-tumor immunologic relationships, there may be concern raised for routinely removing unaffected LNs. Indeed, the extirpation of an immunologic defense organ may alter the host response to the tumor [10].

In the current series, lymphadenectomy was well tolerated, with no reported surgical complications. Additionally, even if this study failed to demonstrate a survival benefit for dogs undergoing lymphadenectomy compared with the nodal observation group, among the operated cases there was a reduced incidence of CMCT progression and new CMCT development. The first observation 
seems to be in line with the previously reported hypothesis that PRL might eliminate a potential neoplastic reservoir, representing at the same time a safe clinical procedure without evident complications. Indeed, in the current study HN1 LNs were considered as uninvolved, but still they contain an increased number of mast cells compared to normal nodes, which could represent a micrometastatic load rather than a reactive mast cell proliferation $[15,27]$.

It must be stressed that, according to our agreement study, $5 \%$ of dogs had a cytologically uninvolved LN, yet an early metastatic disease (HN2) based on histopathology. Also, the cytological slides were analyzed by board-certified clinical pathologists, which is not always performed in clinical practice, at least in our country. As a consequence, the false-negative results may be higher if the LN cytology slides are not sent to experienced clinical pathologists. Additionally, if the LNs are not palpable, they may not undergo cytological evaluation, so the nodal status is often unknown at the time of surgery. Because the therapeutic role of HN2 LN extirpation has been previously demonstrated [7], and due to the lack of complications related to lymphadenectomy $[4,8,11]$, to recommend this additional surgical procedure may not be viewed as unnecessary or harmful. On the contrary, it may provide a clinical benefit, as shown by the reduced incidence of MCT progression found in the current series.

The finding that dogs undergoing lymphadenectomy of clinically uninvolved LN also had a reduced risk of de novo cMCT development is more difficult to explain. The most plausible explanation is that dogs in the OO group were more likely to develop new cMCTs, as predisposed breeds were more represented. More speculatively, it may be hypothesized that quiescent neoplastic cells residing in the regional LN may at some point regain the cell cycles and relocate at distant cutaneous sites, giving rise to overt disease [28]. In the current series, it was not investigated whether the new cMCTs were of the same histological grade and mutational status of the primary tumor, impeding any further comment.

In both groups the median ST was not reached, therefore it cannot be excluded that a survival advantage in either group may emerge with longer follow-ups. A power analysis was not performed but, due to the overall favorable prognosis for dogs with stage I low-grade cMCTs, very few tumor related events are to be expected, thus a very large number of cases would be needed to find a difference.

Several limitations of this study should be noted.

First, dogs did not undergo sentinel LN removal and, as a result, this study may have misdirected their lymphadenectomy in $25-60 \%$ of cases $[11,12]$. As a consequence, it cannot be excluded that the extirpation of the sentinel rather than the regional LN would have improved outcome. Additionally, lymphocenters sometimes consist of more than a single LN. Therefore, it is possible that the entire lymphocenter was not removed during the lymphadenectomy, leaving additional regional LNs behind.

Second, the histological classification of $\mathrm{HNO} / \mathrm{HN} 1$ nodes may have been impacted by the number of sections. Unlike human cancer pathology, there are currently no guidelines in veterinary medicine on how to section a LN and on how many sections need to be examined. In the current study, all LNs were sectioned along the major axis at the level of the hilus; thus, cell aggregates qualifying for HN2 nodal stage may have been missed.

Third, this study suffers the bias which are inherent to retrospective analysis. The surgeon's decision as to whether to perform lymphadenectomy may depend not only on disease characteristics such as stage or histology, but also on the anatomic location, tumor size, or owner's willingness. Consequently, dogs requiring a difficult surgical procedure (including, among others, axillary lymphadenectomy) would find themselves in a no-lymphadenectomy group, whereas those with an easily accessible cMCT and/or regional LN may undergo lymphadenectomy more commonly. Also, even if surgical complications related to lymphadenectomy were not reported, it may be possible that minor sequalae were not documented; also, no quality-of life assessment was carried out, potentially hiding disadvantages of the additional treatment burden.

Additionally, only dogs with completely resected, lowgrade cMCTs were included in the study, and this information is often retrieved only after surgery. Nevertheless, provided that cytologic grading may help predicting the histological grade, due to the high rate of locoregional relapse, high-grade cMCTs will require lymphadenectomy in any case $[4,24,29,30]$.

For the agreement study, samples were not evaluated by all anatomic pathologists involved in the study, possibly biasing the results.

Last, even though the median follow-up time was not significantly different among groups, dogs undergoing $\mathrm{OO}$ were monitored for a median of 813 days versus a median of 763 days for dogs undergoing nodal dissection. It cannot be excluded that, with a longer follow-up in operated dogs, the outcome differences may cancel out.

In conclusion, whether regional dissection of clinically negative LNs should be part of the primary resection for cMCTs remains a problem of legitimate concern.

Our results showed that lack of immediate lymphadenectomy was associated with a higher risk for tumor progression. This preliminary judgement, reinforced by the findings that lymphadenectomy was well tolerated in 
all cases, and that histopathology provides the definitive assessment of the nodal pathological status, may suggest that prophylactic lymphadenectomy is indicated in the management of stage I cMCTs. Larger prospective studies are warranted for generating clinical evidence of this latter hypothesis.

\section{Material and methods}

This retrospective multi-institutional study consisted of an agreement part and a clinical part.

The agreement study was aimed at assessing the consistency of cytology in the correct identification of uninvolved LNs, by testing the concordance between Krick's cytological classification and Weishaar's histological classification in diagnosing a LN as non-metastatic.

The clinical study was aimed at assessing the therapeutic role of PRL of grossly and cytologically unremarkable regional LNs in canine low-grade, completely resected cMCT. To do so, the long-term outcomes of MCT-bearing dogs with cytologically unremarkable and surgically unresected regional LN were compared with those of dogs with a surgically resected and histologically normal or minimal risk regional LN.

The regional LN was defined as the closest $\mathrm{LN}$ in the expected lymphatic drainage, and was identified either by palpation or by ultrasound [31].

Regional LNs were considered cytologically free of metastasis if classified as normal or with reactive lymphoid hyperplasia according to the scheme proposed by Krick [16]. They were considered histologically free of metastasis if classified as HNO or HN1 according to the scheme proposed by Weishaar [15].

\section{Agreement study}

For the agreement study, dogs with low grade cMCTs and cytologically negative (normal/reactive according to Krick's criteria) [16] regional LNs undergoing subsequent lymphadenectomy and histological examination were identified from the same oncology centers participating in the clinical study. Two of the four centers had the cytologic and histologic preparations read out by internal board-certified veterinary anatomic pathologists. The remaining two centers had both cytological and histologic samples submitted to the same private laboratory and were read out by two board-certified clinical pathologists and two anatomic pathologists, respectively. All cytological preparations had been obtained by FNA, with or without ultrasound guidance using $27 \mathrm{G}$ or $25 \mathrm{G}$ needles. Smears were generated from obtained sample material and air-dried, and then stained with May Grünwald-Giemsa. All histological samples were fixed in 10\% neutral-buffered formalin and paraffin-embedded following routine processing. Serial sections were cut and routinely stained with hematoxylin and eosin for histologic evaluation. Replicate sections were stained with toluidine blue or Giemsa to highlight mast cell granules. For each dog, the histological findings were correlated with the cytological findings, in order to evaluate the negative predictive value of cytology in the identification of MCT nodal metastases; i.e., the probability that dogs with a cytological result of an unremarkable LN will have a histologically unremarkable LN as well.

\section{Clinical study}

For this part, the medical records of 4 oncology centers were reviewed from January 1st,2010 to January 1st, 2020 to identify dogs with treatment-naive, firstly occurring, completely resected, low-grade cMCTs. Such dogs were further divided into dogs with [1] non surgically resected regional LNs (OO group) that were cytologically-negative (normal/reactive according to Krick's criteria) [16] or dogs with [2] surgically resected regional LNs (PRL group) that were histologically-negative (HNO/HN1 according to Weishaar's criteria) [15]. Decisions regarding whether to perform OO or PRL were made according to each clinician's discretion.

Tumors were defined as completely resected in the absence of neoplastic cells within $2 \mathrm{~mm}$ from surgical margins, assessed microscopically on sections obtained by the breadloaf-cross method.

To be eligible for recruitment, dogs had to undergo complete staging and surgical excision of the primary cMCT, consisting of wide lateral surgical margins of $3 \mathrm{~cm}$ and one fascial plane deep, and have a minimum follow-up of 180 days. Information on clinical stage was obtained by means of hematological and biochemical analysis, cytological evaluation of the cMCT and regional $\mathrm{LN}$, thoracic radiographs, abdominal ultrasound, and FNA of liver and spleen.

Dogs with recurrent, concurrent multiple, subcutaneous or high-grade MCTs or those with stage II-IV disease were excluded from the study. Also, dogs were excluded if they had received neoadjuvant or adjuvant antitumoral treatment, and if the cMCTs had been removed with incomplete margins.

Background information recorded for each dog included: signalment; cMCT description (location, size, presence of ulceration); clinical substage; date of surgery; local relapse (defined as the cytological evidence of a recurrent $\mathrm{cMCT}$ within $2 \mathrm{~cm}$ from previous scar); nodal relapse (defined as the development of cytologically or histologically-confirmed LN metastases); distant relapse (defined as the occurrence of visceral metastasis); development of de novo cMCTs (defined as the occurrence of a new CMCT at a distant cutaneous site having a different 
regional LN), date of death or last follow-up examination, and cause of death.

The characteristics of relapse (local, nodal and distant) and the survival impact were compared between the $\mathrm{OO}$ and PRL groups.

Regardless of the group, dogs were monitored postoperatively by means of clinical examination, blood testing, and abdominal ultrasound, performed every 3 months during the first year, and every 6 months thereafter. In case of progression of any type, dogs underwent a complete re-staging.

\section{Statistical analysis}

Descriptive statistics were used in the analysis of dogs and tumor characteristics. When appropriate, data sets were tested for normality with the Shapiro-Wilk test. Values were expressed as mean $\pm \mathrm{SD}$ in case of normal distribution, or as median with a range in case of nonnormal distribution.

The distribution of demographic features and possible prognostic variables between the $\mathrm{OO}$ and PRL groups were assessed with Student's T-test (quantitative, parametric variables), the Mann-Whitney $U$ test (quantitative, non-parametric variables) or the Chi-square test/ Fisher's exact test (categorical variables). Chi-square was calculated if all expected cell frequencies were equal to or greater than 5 .

The considered variables included breed (purebred and predisposition to biologically aggressive cMCTs, that is, Shar-pei, Labrador retriever and Golden retriever; and predisposition to low-grade cMCTs, that is, Boxer, French Bouledogue, Weimaraner, Pug, American Staffordshire terrier), age, body weight, sex, neutering status, anatomic site of the primary cMCT (head and neck, trunk and limbs, inguinal/perineal/mammary region and digits), substage, macroscopic tumor largest diameter (measured with a digital caliper), ulceration, and development of new cMCTs.

The influence of the above variables and of lymphadenectomy on cMCT progression and cMCT- related death was investigated with Cox proportional hazards regression analysis. The impact of Krick cytological LN score (normal or reactive - OO group) and Weishaar histological LN score (HN0 or HN1 - PRL group) on cMCT progression and cMCT-related death were also assessed with the same methods. Survival plots were generated according to the Kaplan-Meier product-limit method. For survival analyses, quantitative variables (age, weight and tumor diameter) were dichotomized using the median value as cut-off.

Time to cMCT progression (TTP) was calculated from the date of surgery to the first occurrence of one or more of local, nodal or distant relapse. Dogs with no recurrence or disease progression at the date of the last visit or death were censored.

cMCT-specific survival time (ST) was calculated from the date of surgery to the date of death or to the date of the last visit if death did not occur. Only dogs deceased for cMCT-related causes were considered as events.

Data were analyzed by use of commercial software programs (SPSS Statistics v. 19, IBM, Somers, New York, and Prism v. 5.0, GraphPad, San Diego, California). P values $\leq .05$ were considered significant.

\section{Acknowledgements \\ Not applicable.}

\section{Authors' contributions}

LM conceived the study design, included cases, and was responsible for writing the majority of the manuscript. SS was responsible for data analysis and data interpretation, and prepared tables and Figs. MK was responsible for data interpretation and supervised final edits. WB, UB and MC read out most of the cytologic preparations of the cases included in the study. SS, LA and MG read out most of the histologic preparations of the cases included in the study. LM, $S S, R F, D S, E F, W B, U B, A R, S D M, A F, M G, L A$ and $M C$ all participated in study recruitment and management of patients. All authors have read and approved the manuscript.

\section{Funding}

There was no external funding for this study.

\section{Availability of data and materials}

The datasets generated and/or analyzed during the current study are available from the corresponding author on reasonable request.

\section{Declarations}

Ethics approval and consent to participate

The retrospective study described here involved review of medical records from privately-owned dogs, all receiving care as prescribed by licensed veterinarians. Under such circumstances, no formal review by an Institutional Animal Care and Use Committee is required.

Consent for publication

Not applicable.

\section{Competing interests}

There are no competing interests.

\section{Author details}

'Department of Veterinary Medical Sciences, University of Bologna, Ozzano dell'Emilia, Bologna, Italy. ${ }^{2}$ Department of Pathobiology and Diagnostic Investigation, College of Veterinary Medicine, Michigan State University Veterinary Diagnostic Laboratory, East Lansing, USA. ${ }^{3}$ Department of Small Animal Clinical Science, Institute of Infection, Veterinary and Ecological Science, University of Liverpool, Neston, UK. ${ }^{4}$ Department of Veterinary Medicine, University of Milan, Milan, Italy. ${ }^{5}$ MYLAV Veterinary Laboratory, Rho, Milan, Italy. ${ }^{6}$ Department of Veterinary Sciences, University of Torino, Grugliasco, Torino, Italy.

Received: 27 January 2021 Accepted: 30 September 2021

Published online: 15 October 2021

\section{References}

1. Blackwood L, Murphy S, Buracco P, et al. European consensus document on mast cell tumours in dogs and cats. Vet Comp Oncol. 2012;10(3):e1-e29.

2. Warland J, Amores-Fuster I, Newbury W, et al. The utility of staging in canine mast cell tumours. Vet Comp Oncol. 2014;12(4):287-98. 
3. Worley DR. Incorporation of sentinel lymph node mapping in dogs with mast cell tumours: 20 consecutive procedures. Vet Comp Oncol. 2014;12(3):215-26.

4. Mendez SE, Drobatz KJ, Duda LE, et al. Treating the locoregional lymph nodes with radiation and/or surgery significantly improves outcome in dogs with high-grade mast cell tumours. Vet Comp Oncol. 2020;18(2):239-46.

5. Conzo G, Calò PG, Sinisi AA, et al. Impact of prophylactic central compartment neck dissection on locoregional recurrence of differentiated thyroid cancer in clinically node-negative patients: a retrospective study of a large clinical series. Surgery. 2014;155(6):998-1005.

6. Gambardella C, Patrone R, Di Capua F, et al. The role of prophylactic central compartment lymph node dissection in elderly patients with differentiated thyroid cancer: a multicentric study. BMC Surg. 2019;21881:110-8.

7. Marconato L, Polton G, Stefanello D, et al. Therapeutic impact of regional lymphadenectomy in canine stage II cutaneous mast cell tumours. Vet Comp Oncol. 2018;16(4):580-9.

8. Ferrari $R$, Marconato L, Buracco P, et al. The impact of extirpation of non-palpable/normal-sized regional lymph nodes on staging of canine cutaneous mast cell tumours: a multicentric retrospective study. Vet Comp Oncol. 2018;16(4):505-10.

9. Bodenham D. Malignant melanoma: the problem of lymph-node metastases. Proceeding R Soc Med. 1969;62(November):1090-2.

10. Baum M. Should lymphadenectomy be discarded? I. immunological considerations. J R Coll Surg Edinb. 1973;18(6):351-6.

11. Ferrari R, Chiti LE, Manfredi M, et al. Biopsy of sentinel lymph nodes after injection of methylene blue and lymphoscintigraphic guidance in 30 dogs with mast cell tumors. Vet Surg. 2020;49(6):1099-108.

12. Lapsley J, Hayes GM, Janvier V, et al. Influence of locoregional lymph node aspiration cytology vs sentinel lymph node mapping and biopsy on disease stage assignment in dogs with integumentary mast cell tumors. Vet Surg. 2021;50(1):133-41.

13. Langenbach A, McManus PM, Hendrick MJ, et al. Sensitivity and specificity of methods of assessing the regional lymph nodes for evidence of metastasis in dogs and cats with solid tumors. J Am Vet Med Assoc. 2001;218(9):1424-8.

14. Grimes JA, Secrest SA, Wallace ML, et al. Use of indirect computed tomography lymphangiography to determine metastatic status of sentinel lymph nodes in dogs with a pre-operative diagnosis of melanoma or mast cell tumour. Vet Comp Oncol. 2020;18(4):818-24.

15. Weishaar KM, Thamm DH, Worley DR, et al. Correlation of nodal mast cells with clinical outcome in dogs with mast cell tumour and a proposed classification system for the evaluation of node metastasis. J Comp Pathol. 2014;151(4):329-38.

16. Krick EL, Billings AP, Shofer FS, et al. Cytological lymph node evaluation in dogs with mast cell tumours: association with grade and survival. Vet Comp Oncol. 2009;7(2):130-8

17. Fournier $Q$, Cazzini P, Bavcar S, et al. Investigation of the utility of lymph node fine-needle aspiration cytology for the staging of malignant solid tumors in dogs. Vet Clin Pathol. 2018:47(3):489-500.
18. Thamm DH, Vail DM. Mast cell tumors. In: Withrow SJ, Vail DM, editors. Withrow \& MacEwan's Small Animal Clinical Oncology. Chapter 19. St Louis: Saunders Elsevier; 2007. p. 402-24.

19. Ku C-K, Kass PH, Christopher MM. Cytologic-histologic concordance in the diagnosis of neoplasia in canine and feline lymph nodes: a retrospective study of 367 cases. Vet Comp Oncol. 2017;15(4):1206-17.

20. Kiupel M, Camus M. Diagnosis and prognosis of canine cutaneous mast cell tumors. Vet Clin North Am Small Anim Pract. 2019;49(5):819-36.

21. Weaver DL. Pathology evaluation of sentinel lymph nodes in breast cancer: protocol recommendations and rationale. Mod Pathol. 2010;23(Suppl 2):S26-32.

22. Mutz ML, Boudreaux BB, Royal A, et al. Cytologic comparison of the percentage of mast cells in lymph node aspirate samples from clinically normal dogs versus dogs with allergic dermatologic disease and dogs with cutaneous mast cell tumors. J Am Vet Med Assoc. 2017;251 (4):421-8.

23. Oliveira MT, Campos M, Lamego L, et al. Canine and feline cutaneous mast cell tumor: a comprehensive review of treatments and outcomes. Top Companion Anim Med. 2020;41:100472.

24. Bae S, Milovancev M, Bartels C, et al. Histologically low-grade, yet biologically high-grade, canine cutaneous mast cell tumours: a systematic review and meta-analysis of individual participant data. Vet Comp Oncol. 2020;18(4):580-9.

25. Balch CM, Soong S-J, Bartolucci AA, et al. Efficacy of an elective regional lymph node dissection of 1 to $4 \mathrm{~mm}$ thick melanomas for patients 60 years of age and younger. Ann Surg. 1996;224(3):255-66.

26. Balch CM, Soong S, Ross MI, et al. Long-term results of a multi-institutional randomized trial comparing prognostic factors and surgical results for intermediate thickness melanomas (1.0 to $4.0 \mathrm{~mm}$ ). Ann Surg Oncol. 2000;7(2):87-97.

27. Marconato L, Marchetti V, Francione D, et al. Morphometrical approach for predicting regional lymph node micrometastatic load in canine mast cell tumours: preliminary results. Vet Comp Oncol. 2008;6(3):162-70.

28. Sistigu A, Musella M, Galassi C, et al. Tuning Cancer Fate: Tumor Microenvironment's Role in Cancer Stem Cell Quiescence and Reawakening. Front Immunol. 2020;11:2166.

29. Scarpa F, Sabattini S, Bettini G. Cytological grading of canine cutaneous mast cell tumours. Vet Comp Oncol. 2016;14(3):245-51.

30. Camus MS, Priest HL, Koehler JW, et al. Cytologic criteria for mast cell tumor grading in dogs with evaluation of clinical outcome. Vet Pathol. 2016;53(6):1117-23.

31. Suami H, Yamashita S, Soto-Miranda MA, et al. Lymphatic territories (Lymphosomes) in a canine: an animal model for investigation of postoperative lymphatic alterations. PLoS One. 2013;8(7):1-9.

\section{Publisher's Note}

Springer Nature remains neutral with regard to jurisdictional claims in published maps and institutional affiliations.

Ready to submit your research? Choose BMC and benefit from

- fast, convenient online submission

- thorough peer review by experienced researchers in your field

- rapid publication on acceptance

- support for research data, including large and complex data types

- gold Open Access which fosters wider collaboration and increased citations

- maximum visibility for your research: over 100M website views per year

At $B M C$, research is always in progress.

Learn more biomedcentral.com/submissions 